\title{
Alterações funcionais e biopsicossociais de pacientes com pé diabético
}

Biopsychosocial and adaptive changes in patients with diabetic foot

\author{
O. M. G. Neves ${ }^{1}$; P. S. Nunes ${ }^{2 *}$; F. O. de Carvalho ${ }^{2}$; M. J. M. de Jesus ${ }^{1} ;$ J. A. \\ Aragão ${ }^{3}$; A. A. S. Araujo ${ }^{1,2}$ \\ IPrograma de Pós-Graduação em Ciências Farmacêuticas, Universidade Federal de Sergipe, 49100-000, São \\ Cristóvão-Sergipe, Brasil. \\ ${ }^{2}$ Programa de Pós-Graduação em Ciências da Saúde, Universidade Federal de Sergipe, 49060-100, Aracaju- \\ Sergipe, Brasil. \\ ${ }^{3}$ Departamento de Morfologia, Universidade Federal de Sergipe, 49100-000, São Cristóvão, Sergipe, Brasil.
}

*paulanunes_se@yahoo.com.br

(Recebido em 06 de outubro de 2020; aceito em 18 de março de 2021)

\begin{abstract}
O caráter crônico do pé diabético pode influenciar no desenvolvimento de distúrbios psicológicos, contudo, ainda é preciso compreender as associações do pé diabético às condições biopsicossociais, restrições de funcionalidade e a qualidade de vida destes pacientes. Dessa forma, o objetivo desse caracterizar as alterações funcionais e psicossociais e investigar a qualidade de vida, ansiedade e depressão em pacientes com pé diabético. Para tanto, foi realizado um estudo transversal, por meio de aplicação de questionários (sociodemográfico, Escala de Independência Funcional - Kats, Questionário de qualidade de vida Whoqol-Bref, Escala hospitalar de ansiedade e depressão HADS), em pacientes com diabetes mellitus e com pé diabético atendidos no serviço de cirurgia vascular durante 18 meses em um hospital terciário. Dos pacientes incluídos, 200 possuíam pé diabético, atenderam aos demais critérios de inclusão e aceitaram participar da pesquisa. Cerca de 31\% dos indivíduos estudados apresentaram classificação A de Katz (independência funcional). A qualidade de vida geral determinada pelo Whoqol-Bref foi de 51,1, e entre os domínios, o domínio físico demonstrou a menor média, igual a 32. Esse achado pode ser associado à baixa independência funcional encontrada em $31 \%$ dos pacientes. Foi observada uma prevalência de $29 \%$ de depressão e $23 \%$ de ansiedade nos pacientes com pé diabético. O nível da amputação demonstrou impacto maior que a existência ou não da amputação na qualidade de vida dos pacientes, resultando ainda em uma menor independência funcional. A dificuldade para realizar atividades básicas também influenciou o aumento da prevalência da ansiedade e depressão.
\end{abstract}

Palavras-chave: pé diabético, impacto psicossocial, qualidade de vida.

The chronic character of diabetic foot can influence the development of psychological disorders, however, it is still necessary to understand the associations of diabetic foot with biopsychosocial conditions, functionality restrictions and the quality of life of these patients. Thus, the objective of this study was to characterize the functional and psychosocial aspects and to investigate the quality of life, anxiety and depression in patients with diabetic foot. To this end, a cross-sectional study was carried out, using questionnaires (sociodemographic, Functional Independence Scale (Kats), Quality of life questionnaire Whoqol-Bref, Hospital anxiety and depression scale HADS), in patients with diabetes mellitus and diabetic foot treated at the vascular surgery service for 18 months in a tertiary hospital. Of the patients included, 200 had diabetic foot, met the other inclusion criteria and agreed to participate in the research. About $31 \%$ of the individuals studied had a Katz A classification (functional independence). The general quality of life determined by Whoqol-Bref was 51.1, and among the domains, the physical domain showed the lowest average, equal to 32 . This finding can be associated with the low functional independence found in $31 \%$ of patients. A prevalence of $29 \%$ of depression and $23 \%$ of anxiety was observed in patients with diabetic foot. The level of amputation demonstrated a greater impact than the existence or not of amputation on patients' quality of life, resulting in even less functional independence. The difficulty to perform basic activities also influenced the increase in the prevalence of anxiety and depression.

Keyword: diabetic foot, psychosocial impact, quality of life.

\section{INTRODUÇÃO}

O pé diabético é uma síndrome que se desenvolve como resultado da neuropatia periférica, sendo uma das complicações mais graves da Diabetes Mellitus (DM) [1]. Além das complicações 
que podem ser desenvolvidas por conta da DM, esta doença pode estar associada à comorbidades psicológicas, desencadeando o aumento das taxas de sofrimento emocional e depressão e consequentemente, comprometendo ainda mais a qualidade de vida e funcionalidade dos seus portadores $[2,3]$.

A presença de alterações nos pés de indivíduos com DM tem se mostrado um problema frequente, atingindo a prevalência de $15 \%$ a $25 \%$ desses pacientes nos Estados Unidos nas duas últimas décadas, sendo que este agravo representa $60 \%$ de todas as amputações de membros inferiores não-traumáticos no país [4]. Esta prevalência também pode ser apresentada de acordo com a etiologia do pé diabético, na qual se destaca a neuropatia que varia entre $23-42 \%$, a doença vascular variando entre $9-23 \%$ e entre $5-7 \%$ para lesões nos pés [5, 6]. Além disso, devido ao elevado risco de amputação, pode-se gerar complicações e limitações com grande influência econômica, social e psicológica na vida dos pacientes e de seus familiares [7].

Segundo Pickwel et al. (2017) [8], a qualidade de vida relacionada à saúde é pior em pacientes com diabetes que possuem úlcera ou amputacão, sendo que isto pode resultar em piores desfechos nesses pacientes. Além disso, o risco de depressão em pacientes diabéticos é o dobro daquele em adultos sem diabetes, independentemente do método usado para medir a depressão [9]. Cerca de $20 \%$ a $30 \%$ dos pacientes com diabetes apresentam depressão, o que pode interferir no autocuidado e influenciar na qualidade de vida dos pacientes [10].

Mais de $60 \%$ das amputações não traumáticas de membros inferiores ocorrem em indivíduos diabéticos, sendo que $85 \%$ destas, são precedidas por úlceras nos pés [7,11]. Segundo Adili et al. (2006) [12] e Fejfarová et al. (2014) [13], o caráter crônico do pé diabético pode influenciar no desenvolvimento de distúrbios psicológicos, contudo, ainda é preciso compreender as associações do pé diabético às condições biopsicossociais, restrições de funcionalidade e a própria qualidade de vida. O conhecimento das alterações biopsicossociais e funcionais dos indivíduos com diabetes podem promover uma melhor compreensão das dificuldades que mais influenciam na vida destes pacientes, facilitando a realização de políticas públicas e ações para melhoria do cuidado dos mesmos, bem como dos profissionais que os assistem nos serviços de saúde e academias. Sendo assim, o objetivo desse estudo foi caracterizar as alteraçóes funcionais e psicossociais e investigar a qualidade de vida, ansiedade e depressão em pacientes com pé diabético.

\section{METODOLOGIA}

\subsection{Delineamento do estudo e amostra}

Foi realizado um estudo transversal, composto por 200 indivíduos com pé diabético, internados em uma enfermaria do serviço de cirurgia vascular de um hospital terciário em Sergipe, Brasil. Essa unidade é o único serviço de residência em cirurgia vascular compondo, com outro hospital, os dois únicos serviços de cirurgia vascular do sistema de saúde público de Sergipe, um estado brasileiro que apresentou 201 novos casos de diabetes tipo 2 entre 2002 e 2012 [14].

A coleta de dados foi realizada durante 18 meses por um único pesquisador entre fevereiro de 2016 a agosto de 2017. Nesse período foram recrutados todos os indivíduos internados (864 indivíduos) os quais participaram de uma avaliação clínica prévia para obter diagnóstico preciso de pé diabético. Foram excluídos os indivíduos que possuíam outras patologias vasculares, tais como, varizes, Doença Arterial Periférica (DAP) e Aneurisma de Aorta Abdominal (AAA) (603 pacientes), bem como, indivíduos que apresentassem alterações cognitivas ou psiquiátricas, demência ou enfermidades que impedissem ou dificultassem as respostas aos instrumentos (61 pacientes). Ao final da seleção, participaram do estudo 200 indivíduos com pé diabético (Figura $1)$. 


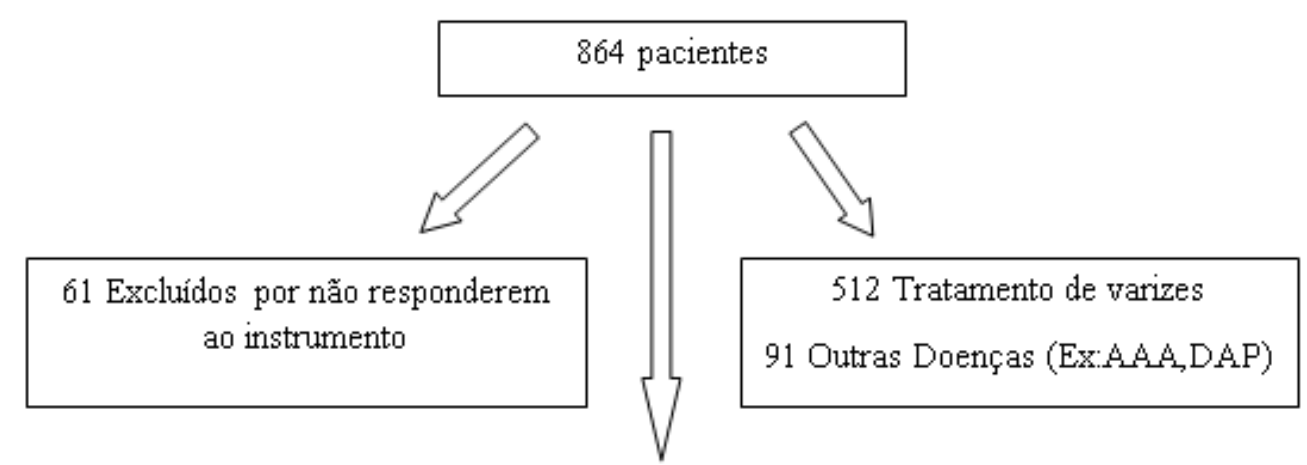

200 individuos selecionados (pé diabético)

Figura 1. Fluxograma do processo de seleção de inclusão e exclusão dos pacientes segundo as comorbidades ou capacidade de preenchimento de instrumentos.

\subsection{Procedimentos de avaliação}

Os indivíduos responderam um questionário de identificação com dados pessoais e sócio demográficos (nome, gênero, idade, procedência, renda familiar mensal, estado civil, grau de escolaridade e ocupação), elaborado pelo próprio autor baseado em estudos prévios contendo identificação e possíveis fatores de risco. Além disso, foi realizada uma triagem sobre comorbidades, sintomatologias clínicas e hábitos de vida (atividade física regular, acompanhamento médico, tabagismo, alcoolismo, hipertensão, diabetes, presença de dor, feridas e/ou amputação prévia). Em seguida, foram aplicados os instrumentos validados no Brasil para avaliação da independência funcional, qualidade de vida e ansiedade e depressão.

\subsubsection{Avaliação da independência funcional}

A funcionalidade dos indivíduos foi avaliada pela Escala Funcional Katz que avalia atividades básicas da vida diária (AVD), referentes ao autocuidado, ou seja, são atividades fundamentais para a manutenção da independência. O escore é obtido de acordo com o desempenho do indivíduo, com pontuação máxima de seis pontos para o indivíduo independente e mínima de 0 para dependência total [15].

\subsubsection{Avaliação dos aspectos psicossociais e qualidade de vida}

A avaliação da qualidade de vida foi realizada utilizando o questionário Who Quality of LifeBref (WHOQOL-Bref),composto por quatro domínios de qualidade de vida, sendo que cada domínio tem por objetivo analisar, respectivamente: a capacidade física, o bem-estar psicológico, as relações sociais e o ambiente onde o indivíduo está inserido. Além desses quatro domínios, o WHOQOL-Bref é composto também por um domínio que analisa a qualidade de vida global [15, 16]. Cada domínio é composto por questões, cujas pontuações das respostas variam entre um e cinco. Os escores finais de cada domínio são calculados por uma sintaxe, que considera as respostas de cada questão que compõe o domínio, resultando em escores finais em uma escala de 4 a 20, comparáveis aos do WHOQOL-100, que podem ser transformados em escala de zero a 100.

Seguindo a mesma linha de estudo utilizada por McDonald et al. (2014) [17], para avaliar o impacto psicossocial, foi utilizado o domínio físico e psicológico do instrumento WHOQOLBref. A subescala de qualidade de vida física consiste em sete questões, que são respondidas em uma escala de um a cinco pontos (1-muito insatisfeito a 5-muito satisfeito) para determinar a 
qualidade de vida física do indivíduo em relação ao período de até duas semanas que antecede a entrevista.

O domínio referente à qualidade de vida psicológica do Whoqol-Bref consiste em seis perguntas que são respondidas em uma escala de um a cinco pontos (1-muito insatisfeito a 5muito satisfeito) para determinar a qualidade de vida referente aos aspectos psicológicos do indivíduo nas duas semanas anteriores.

\subsubsection{Avaliação da ansiedade e depressão}

A Escala Hospitalar de Ansiedade e Depressão (HADS) de Zigmond e Snaith [18] validada no Brasil, possui 14 itens, dos quais sete são voltados para a avaliação da ansiedade (HADS-A) e sete para depressão (HADS-D). Cada um dos seus itens pode ser pontuado de zero a três, compondo uma pontuação máxima de 21 pontos para cada escala. Para a avaliação da frequência da ansiedade e da depressão foram obtidas as respostas aos itens da HADS. Foram adotados os pontos de cortes apontados por Zigmond e Snaith [18] recomendados para ambas as subescalas, HADS-ansiedade e HADS-depressão: sem ansiedade ou depressão de 0 a 8 , com ansiedade ou depressão $\geq 9$.

\subsection{Aspectos Éticos}

Os indivíduos receberam informações sobre os objetivos da pesquisa, instrumentos utilizados e demais procedimentos da coleta de dados. O projeto foi aprovado pelo Comitê de Ética em Pesquisa com Seres Humanos da Universidade Federal de Sergipe (número de protocolo CAAE: 48580115.2.0000.5546) sendo iniciada a coleta de dados após assinatura do Termo de Consentimento Livre e Esclarecido pelos indivíduos.

\subsection{Análise Estatística}

A análise estatística foi realizada utilizando o programa Bioestat 5.3 estabelecendo o intervalo de confiança de $95 \%(\mathrm{p}<0,05)$. Variáveis contínuas foram expressas pela média e desvio-padrão. Os estudos de associação foram realizados utilizando o teste de Qui-quadrado

\section{RESULTADOS}

Os participantes do estudo apresentaram média de idade de 67 anos (com variação de 38 a 91 anos), sendo $54 \%$ do sexo masculino. Foi observado que 56,5\% dos pacientes possuíam união estável, 59,5\% possuíam ensino fundamental, 72\% eram aposentados e 65,5\% tinham renda de até um salário mínimo (Tabela 1). Verificou-se que $81,3 \%$ dos pacientes não realizavam atividade física de forma regular e 64\% realizavam acompanhamento médico de rotina (1-2 consultas: 16,5\%; 3-5 consultas: 46,3\%; mais de cinco consultas: 37,2\%) nos últimos 12 meses. 
Tabela 1: Dados sociodermográficos, comorbidades e fatores de risco dos pacientes com pé diabético.

\begin{tabular}{lll}
\hline Sexo & & Dados sociodermográficos \\
& Feminino & \\
Procedência & Masculino & $46 \%$ \\
& Interior & $54 \%$ \\
& Capital & $65 \%$ \\
Casados & & $35 \%$ \\
& Sim & \\
Escolaridade & Não & $56,55 \%$ \\
& Ensino fundamental & $43,5 \%$ \\
Renda & Não alfabetizado & $59,5 \%$ \\
& & $21,4 \%$ \\
& $<1 S M$ & $65,5 \%$ \\
\hline
\end{tabular}

O tabagismo foi verificado em $37 \%$ da amostra, o alcoolismo em $40 \%$ e a hipertensão arterial sistêmica em $80,7 \%$ dos pacientes. Nesse estudo, $77,4 \%$ dos pacientes claudicavam ou referiam dor em repouso e $76 \%$ eram afetados por feridas atingindo os membros inferiores. A amputação acometeu $56 \%$ dos pacientes estudados sendo que $88,7 \%$ realizaram amputação menor (abaixo do nível do tornozelo) e 11,3\% realizaram amputação maior (perna ou coxa). O comprometimento arterial periférico esteva presente em $70 \%$ desses indivíduos (Tabela 2).

Tabela 2: Comorbidades e possíveis fatores de risco para depressão para os pacientes com pé diabético.

\begin{tabular}{lll}
\hline & Sim & Não \\
\hline Mora sozinho & $9 \%$ & $91 \%$ \\
Acompanhante no hospital & $92 \%$ & $8 \%$ \\
Prática de Atividade física & $19 \%$ & $81 \%$ \\
Até 1 ida ao médico (1 ano)? & $82,3 \%$ & $17,7 \%$ \\
Tabagismo & $37 \%$ & $63 \%$ \\
Etilismo & $40 \%$ & $60 \%$ \\
Possui ferida & $76 \%$ & $24 \%$ \\
Dor em MMI & $77,4 \%$ & $22,6 \%$ \\
Hipertensão arterial & $80,7 \%$ & $19,3 \%$ \\
Amputação prévia & $56 \%$ & $44 \%$ \\
Nível de amputação & $88,7 \%$ (Menor) & $11,3 \%$ (Maior) \\
\hline
\end{tabular}

Realizou-se uma subdivisão dos pacientes com pé diabético sendo feita análise de possíveis fatores de risco em grupos separados pelo gênero, nível de amputação e presença de depressão e ansiedade apresentado na Tabela 3. 
Tabela 3: Fatores de risco segundo grupos subdivididos por depressão, ansiedade, gênero e nível de amputação.

\begin{tabular}{|c|c|c|c|c|c|c|c|c|c|c|c|c|}
\hline \multirow[b]{2}{*}{ Fator de risco } & \multicolumn{2}{|c|}{ Depressivos } & \multicolumn{2}{|c|}{ Ansiosos } & \multicolumn{2}{|c|}{ Feminino } & \multicolumn{2}{|c|}{ Masculino } & \multicolumn{2}{|c|}{$\begin{array}{l}\text { Amputação } \\
\text { Maior }\end{array}$} & \multicolumn{2}{|c|}{ Amputação Menor } \\
\hline & Sim & Não & Sim & Não & Sim & Não & Sim & Não & Sim & Não & Sim & Não \\
\hline Atividade física & $16 \%$ & $84 \%$ & $10,9 \%$ & $89,1 \%$ & $13,3 \%$ & $86,7 \%$ & $23,1 \%$ & $76,9 \%$ & $18,75 \%$ & $81,25 \%$ & $16 \%$ & $84 \%$ \\
\hline Tabagismo & $36,3 \%$ & $63,7 \%$ & $28,9 \%$ & $71,1 \%$ & $25 \%$ & $75 \%$ & $48 \%$ & $52 \%$ & $25 \%$ & $75 \%$ & $36,8 \%$ & $63,2 \%$ \\
\hline Etilismo & $25,5 \%$ & $74,5 \%$ & $33,3 \%$ & $66,4 \%$ & $18,8 \%$ & $81,2 \%$ & $60,2 \%$ & $39,8 \%$ & $25 \%$ & $75 \%$ & $37,8 \%$ & $62,2 \%$ \\
\hline Feridas em MMII & $68 \%$ & $32 \%$ & $75 \%$ & $25 \%$ & $76,5 \%$ & $23,5 \%$ & $75 \%$ & $25 \%$ & $66,6 \%$ & $33,4 \%$ & $78,3 \%$ & $21,7 \%$ \\
\hline Hipertensão & $91 \%$ & $9 \%$ & $84,4 \%$ & $15,6 \%$ & $85,5 \%$ & $14,5 \%$ & $76,8 \%$ & $23,2 \%$ & $81,25 \%$ & $18,75 \%$ & $85 \%$ & $15 \%$ \\
\hline Amputação & $62 \%$ & $38 \%$ & $63,6 \%$ & $36,4 \%$ & $45,5 \%$ & $54,5 \%$ & $64,8 \%$ & $35,2 \%$ & $100 \%$ & $100 \%$ & $100 \%$ & $100 \%$ \\
\hline Ansiedade & $59 \%$ & $41 \%$ & $100 \%$ & $100 \%$ & $24,4 \%$ & $75,6 \%$ & $24 \%$ & $76 \%$ & $50 \%$ & $50 \%$ & $22,1 \%$ & $77,9 \%$ \\
\hline Depressão & $100 \%$ & $100 \%$ & $67,4 \%$ & $32,6 \%$ & $28,8 \%$ & $71,2 \%$ & $27,7 \%$ & $72,3 \%$ & $62,5 \%$ & $37,5 \%$ & $28 \%$ & $72 \%$ \\
\hline \multirow[t]{2}{*}{ Nivel de amputação } & $24,4 \%$ & $75,6 \%$ & $23,3 \%$ & $76,7 \%$ & $19,5 \%$ & $80,5 \%$ & $9 \%$ & $91 \%$ & $100 \%$ & $0 \%$ & $100 \%$ & $0 \%$ \\
\hline & Maior & Menor & Maior & Menor & Maior & Menor & Maior & Menor & Maior & Maior & Menor & Menor \\
\hline
\end{tabular}


A independência funcional para as AVD é classificada como A no índice Katz e foi encontrada em $31 \%$ dos pacientes do estudo. $\mathrm{O}$ índice $\mathrm{C}$ foi o encontrado como o de menor frequência $(1,4 \%)$, demonstrando que $1,4 \%$ dos indivíduos se consideram independentes para todas as atividades básicas de vida, exceto tomar banho. A dependência para pelo menos duas outras funções pôde ser encontrada em $35,1 \%$ dos indivíduos (Tabela 4).

Tabela 4: Classificação da amostra segundo índice Katz, Pé diabético (Pé DM) e nível de amputação (Amp) (Quadro adaptado de Katz, 1963 [15]).

\begin{tabular}{|c|c|c|c|c|}
\hline & Tipo de Classificação & $\begin{array}{c}\text { Pé DM } \\
\text { (geral) } \\
\%\end{array}$ & $\begin{array}{l}\text { Amputação } \\
\text { menor } \\
\%\end{array}$ & $\begin{array}{l}\text { Amputação } \\
\text { maior } \\
\%\end{array}$ \\
\hline A & Independente para todas atividades & 31 & 26,6 & 0 \\
\hline B & $\begin{array}{l}\text { Independente para todas atividades } \\
\text { menos uma }\end{array}$ & 8,5 & 3,4 & 0 \\
\hline $\mathrm{C}$ & $\begin{array}{l}\text { Independente para todas atividades } \\
\text { menos banho e outra. }\end{array}$ & 1,4 & 6,7 & 0 \\
\hline $\mathrm{D}$ & $\begin{array}{l}\text { Independente para todas atividades } \\
\text { menos banho, vestir-se e outra. }\end{array}$ & 2,8 & 10 & 0 \\
\hline $\mathrm{E}$ & $\begin{array}{l}\text { Independente para todas atividades } \\
\text { menos banho, vestir-se ir ao banheiro e } \\
\text { outra adicional }\end{array}$ & 4,2 & 6,7 & 0 \\
\hline $\mathrm{F}$ & $\begin{array}{l}\text { Independente para todas as atividades } \\
\text { menos banho, vestir-se, ir ao banheiro } \\
\text { transferência e outra adicional }\end{array}$ & 10 & 20 & 20 \\
\hline G & Dependente para todas atividades & 7 & 16,6 & 60 \\
\hline Outro & $\begin{array}{l}\text { Dependente em pelo menos duas funções, } \\
\text { mas que não se classificasse em C, D, E e } \\
\text { F }\end{array}$ & 35,1 & 10 & 20 \\
\hline
\end{tabular}

Ao analisar a qualidade de vida geral desses pacientes pelo Whoqol-Bref, foi encontrada uma pontuação média de 51,1. O domínio físico (D1) obteve menor pontuação, apresentando média igual a 32,8 enquanto o domínio com maior pontuação média foi o D3 relações sociais), com média de 70,5 .

A Tabela 5 mostra os resultados do domínio psicológico que aborda relações sociais por meio da avaliação de questões relacionadas à satisfação com a vida sexual, apoio de amigos, além da relação social propriamente dita.

Tabela 5: Estratificação da qualidade de vida segundo pontuação do questionário Whoqol-Bref.

\begin{tabular}{llllll}
\hline \multicolumn{6}{c}{ Pacientes com pé diabético } \\
\hline Domínios & Geral & Amputados & $\begin{array}{l}\text { Não } \\
\text { Amputados }\end{array}$ & $\begin{array}{l}\text { Amputação } \\
\text { menor }\end{array}$ & $\begin{array}{l}\text { Amputação } \\
\text { maior }\end{array}$ \\
\hline D1 (Físico) & $32,8 \pm 17,64$ & $33,22 \pm 14,18$ & $36,36 \pm 22,71$ & $32,23 \pm 0,66$ & $28,12 \pm 0,58$ \\
D2(Psicológico) & $53,5 \pm 19,69$ & $55,43 \pm 14,25$ & $58,56 \pm 13,80$ & $60,21 \pm 0,55$ & $46,25 \pm 0,54$ \\
D3(Rel.Sociais) & $70,5 \pm 21,85$ & $72,47 \pm 14,11$ & $74,06 \pm 13,91$ & $85,32 \pm 0,61$ & $64,06 \pm 0,77$ \\
D4 (Ambiente) & $50,40 \pm 16,80$ & $52,79 \pm 11,82$ & $52,06 \pm 8,86$ & $54,16 \pm 0,46$ & $58,51 \pm 0,58$ \\
QV Geral & $51,25 \pm 15,64$ & $51,78 \pm 21,77$ & $58,56 \pm 22,71$ & $51,63 \pm 0,45$ & $43,75 \pm 0,53$ \\
\hline
\end{tabular}


Foi observado que os pacientes com pé diabético possuíam 23\% (n=46) de ansiedade e 29\% $(n=58)$ de prevalência de depressão. Em relação aos pacientes com algum grau de amputação, foi observado que $27 \%$ dos pacientes amputados foram classificados com algum grau de ansiedade $\mathrm{e}$ $32,4 \%$ desses indivíduos amputados apresentaram depressão. A associação entre depressão e a amputação não se mostrou significativa entre o grupo de pacientes amputados e não amputados $(\mathrm{p}=0,2149)$. Em relação a ansiedade, também não foi observada associação com a amputação $(\mathrm{p}=0,1561)$.

Cerca de 19\% dos pacientes com pé diabético realizavam algum tipo de atividade física semanal. Destes pacientes, aqueles submetidos a algum grau de amputação mostraram redução para $17,5 \%$ $(\mathrm{n}=20)$. Além disso, observou-se que dos pacientes amputados que praticavam atividade física $(n=20), 35 \%$ tinham depressão.

\section{DISCUSSÃO}

A maioria dos pacientes incluídos apresentou baixo grau de escolaridade, com destaque para indivíduos com alguma amputação prévia, público que em sua maioria pertencia ao sexo masculino. No estudo de Mcdonalds et al. (2014) [17] também foi identificado um menor grau de escolaridade em indivíduos com algum grau de amputação (56\% com 12 anos ou menos de escolaridade). A baixa taxa de escolaridade, bem como a influência do gênero são fatores que podem dificultar a busca ao atendimento médico de rotina, impossibilitando o controle da doença. De acordo com Gomes et al. (2011) [19], o imaginário social que vê o homem como ser invulnerável, aliado a desinformação acaba contribuindo para que ele se cuide menos e se exponha mais a situações de risco.

Nesse contexto, foi interessante notar que o tabagismo e o alcoolismo podem ter influenciado no aumento do nível de amputação. Em um estudo realizado na Índia, foi observado que os pacientes diabéticos que eram fumantes e/ou alcoólistas crônicos sofreram mais amputações do que os pacientes que não tinham dependência dessas substâncias [20]. Isso demonstra a necessidade da mudança de estilo de vida em pacientes com diabetes para melhor conviver com a doença e buscar uma expectativa de vida ainda maior [21].

Mantovani et al. (2017) [22] avaliaram a relação entre amputação e fatores de risco em pessoas com diabetes mellitus e demonstraram que os principais fatores preditivos de risco para amputação nessa população, foram a presença de úlcera e o tabagismo. Pacientes diabéticos que fumam apresentam maiores riscos de complicações sérias, como fluxo sanguíneo deficiente nas extremidades, doença arterial periférica e neuropatia periférica, o que pode gerar infecções, úlceras e consequentemente possíveis amputações.

Pode-se ressaltar ainda que o consumo de quantidades moderadas de álcool (duas a quatro doses por dia) interfere no controle do açúcar no sangue de pacientes diabéticos e aumenta o risco de neuropatia periférica e subsequente desenvolvimento de úlcera do pé diabético com mau prognóstico, o que pode levar ao aumento da incidência de amputação [23].

Em relação à prática de atividade física, os pacientes do gênero masculino com pé diabético foram mais adeptos quando comparados ao público feminino. A atividade física regular é incentivada em pacientes com diabetes em geral, a fim de promover o controle glicêmico, reduzir o risco cardiovascular, melhorar a dor e a qualidade de vida. Coffey et al. (2019) [24], realizaram uma metanálise qualitativa com pacientes diabéticos com risco de ulceração e observaram presença de dor e redução de mobilidade nos indivíduos que foram menos adeptos à prática de atividade física. No entanto, em pacientes com risco de ulceração, alguns estudos têm demonstrado, que a realização de atividade física pode aumentar as chances de desenvolvimento de úlceras podendo influenciar negativamente na qualidade de vida dos mesmos [25, 26].

Sobre a independência funcional foi verificado que menos de um terço dos indivíduos do presente estudo atingiram a independência funcional (classificação A de Katz), o que pode indicar que o diabetes ou amputação podem causar mais dependência funcional. Nesse sentido, Ramos et al. (2017) [27] observaram que a prevalência do diabetes mellitus mostrou-se maior entre os idosos classificados como dependentes pela escala de Katz, em relação aos independentes. Um achado 
interessante foi encontrado por Ramos et al. (2017) [27], no qual aproximadamente um quarto dos paciente, apesar de dependentes funcionalmente, classificaram sua saúde como excelente.

Ainda nesse sentido, Tavares et al. (2021) [28], observaram influencia da depressão na piora funcional enquanto Leite et al. (2012) [29], em um estudo que avaliou a mobilidade funcional de idosos diabéticos e não diabéticos, constataram que o grupo com diabetes tiveram pior desempenho nos testes, o que pode gerar um maior risco de quedas. Dessa forma, pode-se inferir que o grau de independência funcional nos pacientes com pé diabético é baixo, devido ao grande número de complicações associadas, como déficit de equilíbrio corporal, gerando uma marcha instável e mais lentificada [30, 31].

Ao mensurar o impacto psicossocial, McDonald et al. (2014) [17] utilizaram o domínio físico e psicológico. No presente estudo a média encontrada no domínio físico para pacientes amputados foi menor do que a pontuação encontrada em pacientes amputados no trabalho de McDonald et al. (2014) [17]. Ainda foi verificado, neste estudo, menores valores no domímio físico em indivíduos com diabetes sem amputação quando comparado aos valores encontrados por McDonald et al. (2014) [17]. O presente estudo teve uma amostra de amputados cerca de 2 vezes maior e foi realizado em um pais com índices socioeconômicos mais baixos e maior deficiência do serviço público de saúde, fatores que podem justificar esses resultados. Vale reesaltar porém, que ambos os estudos evidenciam o impacto significativo que a amputação pelo diabetes pode causar no disturbio de imagem do indivíduo, podendo ser demonstrado por variação negativa proporcional dos domínios físicos e psicológicos. Foi observado que 32,4\% dos pacientes com pé diabético apresentavam depressão e $27 \%$ apresentavam algum nível de ansiedade. Uma maior prevalência de depressão foi verificada por Ahmad et al. (2018) [32], que estudaram 260 paciente com pé diabético entre 2015 e 2016 na Jordânia, encontraram uma prevalência de depressão de 39,6\% e de ansiedade de 37,7\%. Fejfarová et al. (2014) [13] observaram uma prevalência de depressão e ansiedade de $83.2 \%$ a $89.6 \%$, respectivamente, em uma amostra de 104 pacientes com pé diabético.

O uso de diferentes escalas para avaliar depressão (Escala de depressáo geriatrica (GDS) e Escala hospitalar de ansiedade de depressão (Hads) além da amostra cerca de 2 vezes maior no presente estudo, podem justificar essas alteraçoes. Em outro estudo realizado em 2011 por Salomé et al. (2011) [33], verificaram que $98 \%$ dos pacientes com pé diabetico que apresentavam úlceras possuiam algum sintoma de depressão, sendo que $64 \%$ apresentaram depressão moderada e $10 \%$ depressão grave. Uma possível justificativa está na necessidade de cuidados com ferimentos desde curativos ao uso de medicacoes com efeitos adversos, além de outros posséveis sintomas associados as úlceras que podem influenciar aumentando depressão e ansiedade. Outra hipótese que pode ter relação com o percentual mais baixo de depressão e ansiedade neste estudo quando comparado aos demais é que, diferente dos estudos anteiores, neste, cerca de $77 \%$ dos pacientes possuíam dor em algum membro, sendo que $21 \%$ utilizavam antidepressivos tricíclicos para tratamento de dor.

No presente estudo foi observado uma maior prevalência de depressão e ansiedade em pacientes com amputação prévia e pé diabético, no entanto, sem diferença estatisticamente significante. Destes pacientes amputados, os pacientes submetidos à amputação menor apresentaram valores mais baixos nas escalas de avalaição de ansiedade e depressão em relação aos pacientes com nível elevado de amputação. Isso nos leva a crer que o nível ou altura da amputação acaba influenciando mais do que apenas a existência da mesma. No estudo de Williams et al. (2010) [34], verificou-se que a depressão em pacientes diabéticos amputados foi associada à amputações maiores (níveis transtibiais e transfemorais), enquanto que nos indivíduos submetidos a amputações menores (abaixo do tornozelo) não houve correlação estatisticamente significativa, o que pode justificar o resultado também não significativo do presente estudo, onde a grande maioria das amputações (85\%) eram em níveis mais baixos. Williams et al. (2010) [34], ainda concluíram que a depressão diagnosticada em pacientes diabéticos aumenta o risco de amputação em níveis mais altos, o que pode gerar maior dependência funcional e pior qualidade de vida. Além disso, em outro estudo realizado por Yilmaz et al. (2016) [35], 135 pacientes amputados foram avaliados quanto a presença de ansiedade usando o mesmo instrumento deste estudo (HADS) sendo que o grupo de amputação transfemoral apresentaram escores mais altos em comparação com aqueles do grupo transtibial ( $p$ $<0,05)$.

Quando abordamos independência funcional relacionada ao nível de amputação, podemos observar que nesse estudo, todos pacientes possuíam mais de 35 anos e, a média geral de 
independência para todas atividades foi atingida por $31 \%$ dos pacientes com pé diabético (geral) sendo 26,6\% nos pacientes com amputação menor e $0 \%$ de independência em pacientes com amputação maior. Ylmaz et al. (2016) [35], estudando independência funcional e utilizando índice Katz observou diferença significativa apenas no grupo com amputação com idade superior a 35 anos. Cox e Weaver (2011) [36] avaliaram 87 pacientes com diabetes e observou que dentre os participantes do estudo, os pacientes amputados abaixo do joelho registraram escores mais altos de QV ( $p<0,05)$ e independência funcional $(\mathrm{p}<0,0001)$ em comparação aos amputados acima do joelho. Tais resultados reforçam ainda mais, a influência do nível de amputação na qualidade de vida e independência funcional, sendo o nível de amputação um fator mais impactante do que a presença ou ausência da amputação.

Apesar deste estudo ser um delineamento transversal e de possuir muitos instrumentos, o que pode ter tornado cansativa a participação dos pacientse, foram abordados inúmeros e possíveis fatores de risco para depressão e ansiedade em pacientes com pé diabético, além de demonstrar a necessidade do conhecimento dos aspectos biopsicosociais e da possível influência na limitação funcional, evidenciando assim a necessidade de um diagnóstico precoce com um olhar mais sensível associado a multidisciplinariedade para realização de uma terapêutica adequada de complicações biopsicossociais com uma reabilitação efetiva.

\section{CONCLUSÃO}

Podemos concluir que o gênero masculino e baixo nível de escolaridade associado ao etilísmo e tabagismo são importantes fatores associados a amputaçao.

Ainda podemos concluir que as amputações acima do nível do tornozelo têm relação com as alteraçoes psicossociais e funcionais dos indivíduos com pé diabético, estando associada a uma maior prevalência de depressão e pior qualidade de vida.

\section{AGRADECIMENTOS}

Os autores agradecem ao Conselho Nacional de Desenvolvimento Científico e Tecnológico $(\mathrm{CNPq})$, a Fundação de Apoio à Pesquisa e à Inovação Tecnológica (FAPITEC/SE) e a Coordenação de Aperfeiçoamento de Pessoal de Nível Superior (CAPES) pelo apoio no desenvolvimento da pesquisa.

\section{REFERÊNCIAS BIBLIOGRÁFICAS}

1. Rubin RR, Peyrot M. Psychological issues and treatments for people with diabetes. J Clin Psychol. 2001;57(4):457-478, doi: 10.1002/jclp.1041.

2. Ali S, Stone MA, Peters JL, Davies MJ, Khunti K. The prevalence of comorbid depression in adults with Type 2 diabetes: a systematic review and meta-analysis. Diabet Med. 2006;23(11):1165-1173, doi: 10.1111/j.1464-5491.2006.01943.x.

3. Strauss MB, Moon H, La S, Craig A, Ponce J, Miller S. The incidence of confounding factors in patients with diabetes mellitus hospitalized for diabetic foot ulcers. Wounds. 2016;28(8):287-294.

4. Kalish J, Hamdan A. Management of diabetic foot problems. J Vasc Surg. 2010;51(2):476-486, doi: 10.1016/j.jvs.2009.08.043.

5. Wild S, Roglic G, Green A, Sicree R, King H. Global prevalence of diabetes: estimates for the year 2000 and projections for 2030. Diabet Care. 2004;27(5):1047-1053.

6. Lipsky BA, Berendt AR, Cornia PB, Pile JC, Peters EJ, Armstrong D, et al. Infectious Diseases Society of America clinical practice guideline for the diagnosis and treatment of diabetic foot infections. Clin Infec Dis. 2012;54(12):e132-e173, doi: 10.1093/cid/cis346.

7. Jesus-Silva SG, Oliveira JP, Colepicolo Brianezi MH, Moraes Silva MA, Krupa AE, Souza Cardoso R. Analysis of risk factors related to minor and major lower limb amputations at a tertiary hospital. J Vasc Bras. 2017;16(1):16-22, doi: 10.1590/1677-5449.008916.

8. Pickwell K, Siersma V, Kars M, Apelqvist J, Bakker K, Edmonds M, Piaggesi A. Minor amputation does not negatively affect health related quality of life as compared with conservative treatment in patients 
with a diabetic foot ulcer: An observational study. Diabetes Metab Res Rev. 2017;33:e2867, doi: 10.1002/dmrr.2867.

9. Vileikyte L, Shen BJ, BrownS, BoultonAJ, Kirsner R, Reeves N, Crews RT. Depression, physical activity, and diabetic foot ulcer healing. Diabetes. 2017;66(Suppl):A168.

10. Sales-Peres SHDC, Guedes MDFS, Sá LM, Negrato CA, Lauris JRP. Estilo de vida em pacientes portadores de diabetes mellitus tipo 1: uma revisão sistemática. Ciênc Saúde Col. 2016;21(4):1197-1206, doi: 10.1590/1413-81232015214.20242015.

11. Almeida SAD, Silveira MM, Santo PFDE, Pereira RDC, Salomé GM. Avaliação da qualidade de vida em pacientes com diabetes mellitus e pé ulcerado. Rev Bras Cir Plast. 2013;28(1):142-146, doi: $10.1590 /$ S1983-51752013000100024.

12. Adili F, Larijani B, Haghighatpanah M. Diabetic Patients. Ann N Y Acad Sci. 2006;1084(1):329-349, doi: 10.1196/annals.1372.016.

13. Fejfarová V, Jirkovská A, Dragomirecká E, Game F, Bém R, Dubský, M, et al. Does the diabetic foot have a significant impact on selected psychological or social characteristics of patients with diabetes mellitus? J Diabetes Res. 2014;2014:371932, doi: 10.1155/2014/371938.

14. Filho ACAA, Almeida PD, de Araújo AKL, Sales IMM, de Araújo TME, da Rocha SS. Epidemiological profile of Diabetes Mellitus in a northeastern brazilian state. Revista Online de Pesquisa: Cuidado é Fundamental. 2017;9(3):641-647, doi: 10.9789/2175-5361.2017.v9i3.641.

15. de Oliveira LFS, Wanderley RL, de Medeiros MMD, de Figueredo OMC, Pinheiro MA, Garcia RCMR, Cavalcanti YW. Health-related quality of life of institutionalized older adults: influence of physical, nutritional and self-perceived health status. Arch Gerontology Geriatric. 2021;92:104278, doi: 10.1016/j.archger.2020.104278.

16. Kalfoss MH, Reidunsdatter RJ, Klöckner CA, Nilsen M. Validation of the WHOQOL-Bref: psychometric properties and normative data for the Norwegian general population. Health and Quality of Life Outcomes. 2021;19(1):1-12, doi: 10.1186/s12955-020-01656-x.

17. McDonald S, Sharpe L, Blaszczynski A. The psychosocial impact associated with diabetes-related amputation. Diabet Med. 2014(11);31:1424-1430, doi: 10.1111/dme.12474.

18. Ogawa M, Watanabe Y, Motegi T, Fukui N, Hashijir, K, Tsuboya R, Someya T. Factor structure and measurement invariance of the hospital anxiety and depression scale across the peripartum period among pregnant japanese women. Neuropsychiatri Dis Treat. 2021;17:221-227, doi: 10.2147/NDT.S294918.

19. Gomes R, Moreira MCN, Nascimento EFD, Rebello LEFDS, Couto MT, Schraiber LB. Os homens não vêm ausência e/ou invisibilidade masculina na atenção primária. Ciên Saúde Col. 2011;16(Supl 1):983992, doi: 10.1590/S1413-81232011000700030.

20. Pal B, Raveender N, Sudipta P. A study on the impact of smoking and alcoholism as determinant factors in the prognosis and outcome of diabetic foot ulcer disease. Int J Res Med Sci. 2016;4(5):1720-1724, doi: 10.18203/2320-6012.ijrms20161257.

21. Gois CO, Lima SVMA, dos Santos ACFS, Melo HNAL. Perfil dos portadores de diabetes mellitus atendidos em farmácias particulares de Sergipe, Brasil. Sci Plena. 2017;13(11):1-7, doi: 10.14808/sci.plena.2017.117501.

22. Mantovani AM, Fregonesi CE, Palma MR, Ribeiro FE, Fernandes RA, Christofaro DG. Relationship between amputation and risk factors in individuals with diabetes mellitus: a study with Brazilian patients. Diabet Met Synd: Clin Res Rev. 2017;11(1):47-50, doi: 10.1016/j.dsx.2016.08.002.

23. Kaplan LM. American Diabetes Association. Standards of medical care in diabetes. Diabet care. 2005;28(1):S4.

24. Coffey L, Mahon C, Gallagher P. Perceptions and experiences of diabetic foot ulceration and foot care in people with diabetes: A qualitative meta-synthesis. International Wound Journal. 2019;16(1):183-210, doi: 10.1111/iwj.13010.

25. Vedhara K, Beattie A, Metcalfe C, Roche S, Weinman J, Cullum N, et al. Development and preliminary evaluation of a psychosocial intervention for modifying psychosocial risk factors associated with foot reulceration in diabetes. Behav Res Ther. 2012;50(5):323-332, doi: 10.1016/j.brat.2012.02.013

26. Silva MCA, Pereira DS, Almeida, CDS, Venâncio MIL. Pé diabético e avaliação do risco de ulceração. Rev Enferm Ref. 2014;4(1):153-161, doi: 10.12707/RIII12166.

27. Ramos SPS, Marques OR, Ramos APP, Borba, VOT, Aguiar AKA, Carréra CLM, et al. Fatores associados ao diabetes em idosos assistidos em serviço ambulatorial especializado geronto-geriátrico. Rev Bras Geriatr Gerontol. 2017;20(3):363-373, doi: 10.1590/1981-22562017020.160145.

28. Tavares JPDA, Nunes LANV, Grácio JCG. Pessoa idosa hospitalizada: preditores do declínio funcional. Rev Latino-Americana de Enfer. 2021;29:e3399, doi:10.1590/1518-8345.3612.3399.

29. Leite MAM, Nunes CEA, Martins AMR, Faccenda O, Campos OMA. Avaliação funcional de idosos com déficit cognitivo. Acta Paul Enferm.2012;25(3):358-363, doi:10.1590/S0103-21002012000300007 
30. Petrofsky JS, Cuneo M, Lee S, Johnson E, Lohman E. Correlation between gait and balance in people with and without Type 2 diabetes in normal and subdued light. Med Sci Monit. 2006;12(7):CR273-81.

31. Hong X, Chen X, Chu J, Shen S, Chai Q, Lou G, Chen L. Multiple diabetic complications, as well as impaired physical and mental function, are associated with declining balance function in older persons with diabetes mellitus. Clin Interv Aging. 2017 Jan;12:189-195, doi: 10.2147/CIA.S123985.

32. Ahmad A, Abujbara M, Jaddou H, Younes NA, Ajlouni K. Anxiety and depression among adult patients with diabetic foot: prevalence and associated factors. J Clin Med Res. 2018;10(5):411-418, doi: $10.14740 /$ jocmr3352w.

33. Salomé GM, Pellegrino SDM, Blanes L, Ferreira LM. Self-esteem in patients with diabetes mellitus and foot ulcers. J Tissue Viability. 2011;20(3):100-106, doi: 10.1016/j.jtv.2010.12.004.

34. Williams LH, Rutter CM, Katon, WJ, Reiber GE, Ciechanowski P, Heckbert SR, et al. Depression and incident diabetic foot ulcers: a prospective cohort study. Am J Med. 2010;123(8):748-754, doi: 10.1016/j.amjmed.2010.01.023.

35. Ylmaz M, Gualabi D, Kaya I, Bayram E. The effect of amputation level and age on outcome: an analysis of 135 amputees. Eur J Orthop Surg Traumatol. 2016;26:107-112, doi: 10.1007/s00590-015-1709-z.

36. Cox PSL, Weaver SR. Life after lower extremity amputation in diabetics. West Indian Med J. 2011;60(5):536-540. 